\title{
NOTE ON ELECTRICAL CONDUCTION IN METALS AT LOW TEMPERATURES ${ }^{1}$
}

\author{
By Francis B. Silsbee, Assistant Physicist
}

The experiments of $\mathrm{H}$. Kamerlingh Onnes on the resistivity of metals at liquid helium temperatures have shown that certain metals possess an enormously increased conductivity when the temperature, current density, and magnetic field are less than certain critical values. It is the purpose of this paper to point out that a definite relationship is to be expected between the values of critical current and critical field, and that this relation is in agreement with the experimental data available.

The present state of our experimental knowledge of this subject is somewhat as follows: Certain metals-mercury, tin, and lead-at the very low temperatures obtainable in a bath of liquid helium show a very greatly increased electrical conductivity, to which Onnes has given the name "superconductivity." The actual resistivity of the metal in this state is too small to measure but has been shown to be less than $2 \cdot 10^{-11}$ times the resistivity at $\mathrm{O}^{\circ} \mathrm{C}^{2}$ As the temperature of any of these metals is lowered from room temperature the resistance decreases uniformly with the normal coefficient of about 0.4 per cent per degree until the temperature is very low, when the rate of decrease becomes for a time less rapid. At a certain critical temperature, however $\left(4^{\circ} .2 \mathrm{~K}\right.$ for mercury, $3 .^{\circ} 8 \mathrm{~K}$ for tin, and $6^{\circ} \mathrm{K}$ for lead), ${ }^{3}$ there is a

1 This note, with some modifications, was published in the Journal of the Washington Academy of Sciences, 6, p. 597; October, rgr6.

${ }^{2}$ Kon. Akad. v. Weten., Amsterdam, XVII, r, p. 280.

${ }^{8}$ Communications from Leiden Laboratory No. ${ }_{3} 33$, pp. 7, 52, 60 . 
sudden break in the curve connecting resistance and temperature, and within a temperature range of a few hundredths of a degree the resistance drops from about $10^{-3}$ times its value at $0^{\circ} \mathrm{C}$ to less than $1 \mathrm{O}^{-10}$ times the same value. Other metals, such as gold, silver, platinum, iron, etc., do not show this phenomenon, and their resistivity tends to approach a constant value as the temperature is lowered to the lowest value $\left(I^{\circ} .6 \mathrm{~K}\right)$ at which such measurements have been made. The critical temperature at which the change occurs is very definite when the current used to measure the resistance is small, but when the measuring current is very large the critical temperature is found to be definitely lower. Conversely, if the temperature of the bath be held constant some degrees below the critical value and the current be increased a certain "threshold" value of current will be found at which the resistance suddenly appears. ${ }^{4}$ The lower the temperature the greater the value of the critical current.

It is further found that when a superconductor is placed in a weak magnetic field it remains superconducting, but that as the field is increased the normal resistance appears suddenly at a certain critical value of the magnetic field, and for still higher values of field increases slowly with the field. ${ }^{5}$ The critical value is slightly less when the field is transverse to the direction of current flow than when it is longitudinal, but the difference is not great.

The particular point which is the subject of this note is that the "threshold" value of current is that at which the magnetic field due to the current itself is equal to the critical magnetic field. In other words, the phenomenon of threshold current need not be regarded as a distinct phenomenon to be explained by heating, etc., but is a direct result of the existence of the phenomenon of threshold magnetic field.

In case the specimen is in the form of a flat coil of wire it is evident that the inner turns are in a magnetic field, due to the current in the other turns, which is very similar to that due to an entirely external electromagnet. Consequently, when upon increasing the current this field reaches the critical value, first the inner turns will become resisting and, as the current is increased, more and more of the wire will cease to be superconducting. Because of the enormous factor by which the conductivity decreases from the superconducting to the normal state most of this decrease will take place when only a small fraction of a turn of the coil ceases to be superconducting. Owing to the cumulative 
effect of the successive turns the field produced by a given current is much greater in the coil than in the same wire when straight and consequently the current required to give the critical field strength will be much less. This is verified by the results of Onnes on coils of lead and tin ${ }^{6}$ wire, which showed critical currents one-fifteenth and one-eighth, respectively, of those for the same wire when straight. No attempt has yet been made to measure the gradual further increase of resistance which would be expected on this theory as the current is further increased and more and more turns become resisting.

In the case of a straight wire of circular section the effect to be expected is rather more complicated. Consider a superconducting wire of radius $r_{0}$ carrying a current $I$, uniformly distributed over the cross section. The magnetic field intensity $H$ at any point distant $r$ from the axis but inside the wire is given by

$$
H=\frac{2 I r}{r_{0}^{2}}
$$

and that at the surface of the wire by

$$
H_{\mathrm{o}}=\frac{2 I}{r_{\mathrm{o}}}
$$

If the current be increased to a value slightly greater than $\frac{H_{\mathrm{c}} r_{0}}{2}$, where $H_{\mathbf{c}}$ is the critical-field intensity for the material, the outermost layer of the wire will become resisting. Since this layer is shunted by the superconducting core, the whole current will tend to flow in this core. This, however, would make the field at the edge of the core even greater than that above computed since by equation (2) the field varies inversely as the external radius.

The system is, therefore, unstable, and the current will shift suddenly to a new distribution. This distribution will depend on the exact form of the relation connecting resistivity with magnetic field, and if this relation were known the current distribution might be computed from the usual electromagnetic equations.

If it be assumed, as a special case, that the resistivity changes by a large factor $k$ at a definite field intensity $H_{\mathrm{c}}$, then for currents larger than the critical value there is a superconducting core, which, however, has such a small radius that only a negligible part of the total current flows in it, while the outermost portion becomes resisting. ${ }^{7}$ Prof. Langevin has kindly sent the author a complete

\footnotetext{
${ }^{6}$ Leiden Comm. No. 133, pp. 57, 60.

7 As printed in the Journal of the Washington Academy of Sciences only these two layers were mentioned. This is, therefore, incorrect as noted below.
} 
mathematical solution of this problem, which shows that between these regions there is a third layer in the cross section of the wire in which the current density varies so as to keep the magnetic field at the value $H_{c}$ throughout the layer. Prof. Langevin finds that the radius of the surface separating the intermediate region from the small superconducting core is

$$
r_{1}=r_{0} \frac{2}{k} \frac{\mathrm{I}}{\left[\frac{I}{I_{\mathrm{c}}}+\sqrt{\left(\bar{I}_{I_{\mathrm{c}}}\right)^{2}-\mathrm{I}}\right]}
$$

and that the radius of the surface separating the intermediate and outer layers is

where

$$
r_{2}=r_{0} \frac{I}{\left[\frac{I}{I_{\mathrm{c}}}+\sqrt{\left(\frac{I}{I_{\mathrm{c}}}\right)^{2}-\mathrm{I}}\right]}
$$

$$
\begin{aligned}
I & =\text { total current. } \\
I_{\mathrm{c}} & =\frac{H_{\mathrm{c}} r_{0}}{2}=\text { critical current. }
\end{aligned}
$$

The magnetic field in the three regions is as follows:

In the superconducting core, $H<H_{\mathrm{c}}$.

In the intermediate layer, $H=H_{\mathrm{c}}$.

In the outer layer, $\quad H>H_{\mathrm{c}}$.

The result of this distribution on the resistance of the wire as a whole is to cause a sudden increase of resistance by a factor $\frac{k}{2}$ as the current passes the critical value and a further increase, at first rapid and then slower, as the resistance approaches asymptotically to $k$ times its original value.

For any other relation between resistivity and field there would be a corresponding current distribution. In general the abruptness of the increase of resistance with current would be similar to that of the increase of resistivity with field.

Owing to the great experimental difficulties of working at these extreme temperatures, the data available for an experimental verification of this theory are rather scanty. The following table contains in condensed form the observed values of threshold current for various wires at different temperatures as published by the Leiden Laboratory. Since the threshold values depend considerably on temperature, a comparison is possible only when observations were made on two wires at the same temperature, and the table contains the results of practically all such observations published. ${ }^{8}$ 
In the last column is given the maximum value of magnetic field in any part of the conductor - that is, the field at the surface of a straight wire or at the inner turns of a coil (the computations for the latter case being only approximate)-due to its own threshold current. It is seen from the table that at each temperature this magnetic field is much more nearly a constant of the material than either the current or current density. In the case of mercury the effect of a magnetic field on the resistance in the superconducting state has not been measured. For tin the threshold value at $2^{\circ} \mathrm{K}$. is about 200 gausses, which is in good agreement with the slightly larger values computed from the threshold current corresponding to a slightly lower temperature. In the case of lead the agreement of the observed critical field (600 gausses at $4^{\circ} \mathrm{K}$.) with the computed values is not so good, particularly in the case of the straight wire. Any discrepancy here, however, is easily explained by the possibility (frequently referred to by Onnes) of the existence of thin spots in the wire where the field intensity would be much greater for a short length.

Critical Values of Current for Various Metals and Temperatures

[From data by H. K. Onnes]

MERCURY

\begin{tabular}{|c|c|c|c|c|}
\hline Temperature, degrees $\mathbf{K}$ & Area & $\begin{array}{c}\text { Threshold } \\
\text { current }\end{array}$ & $\begin{array}{l}\text { Threshold- } \\
\text { current } \\
\text { density }\end{array}$ & $\underset{\text { magnetic }}{\text { field }}$ \\
\hline & $\mathrm{mm}^{2}$ & amp. & $\mathrm{amp} . / \mathrm{mm}^{2}$ & gausses \\
\hline \multirow[t]{4}{*}{$4.1 \ldots$} & 0.0016 & 0.17 & 107 & 15 \\
\hline & .0025 & .17 & 69 & 12 \\
\hline & .0055 & .23 & 42 & 11 \\
\hline & .0055 & .32 & 58 & 15 \\
\hline \multirow[t]{4}{*}{ 3.5.. } & .0016 & 1.00 & 625 & 89 \\
\hline & .0025 & 1.07 & 427 & 76 \\
\hline & .004 & $>1.04$ & $>260$ & $>59$ \\
\hline & .0052 & .78 & 151 & 39 \\
\hline
\end{tabular}

TIN. $\mathrm{H}_{\text {crit. }}=200$ at $2^{\circ} \mathrm{K}$

\begin{tabular}{|c|c|c|c|c|}
\hline $1.6, \ldots \ldots \ldots, \ldots$ & $\begin{array}{l}.0143 \\
.0143\end{array}$ & $\begin{array}{l}1.0 \\
8.0\end{array}$ & $\begin{array}{r}70 \\
560\end{array}$ & $\begin{array}{l}a 430 \\
b 240\end{array}$ \\
\hline
\end{tabular}

LEAD. $\mathrm{H}_{\text {crit. }}=600$ at $4^{\circ} .2 \mathrm{~K}$

\begin{tabular}{|c|c|c|c|c|}
\hline 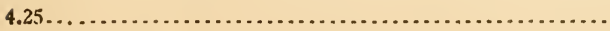 & .025 & 9 & 680 & $b 385$ \\
\hline & .014 & $>4$ & $>300$ & $c>110$ \\
\hline & .014 & .6 & 41 & a 375 \\
\hline  & .014 & .84 & 60 & a 550 \\
\hline & .014 & 11.1 & 790 & b 330 \\
\hline
\end{tabular}


Further experiments which immediately suggest themselves are measurements on the critical magnetic field for mercury. The relation here advanced would indicate a critical field of only about 15 gausses at $4: 1 \mathrm{~K}$ and less than 100 gausses at $3: 6 \mathrm{~K}$. It would also be of interest to observe the threshold value of current when the material is in very thin films. In this case for a given section of material the magnetic field resulting from a given current density is less than in the case of a straight wire, and the threshold-current density would consequently appear larger.

The theories thus far proposed to account for superconductivity by Onnes, ${ }^{9}$ Lindemann, ${ }^{10}$ and Thomson ${ }^{11}$ do not specifically indicate the existence of a critical magnetic field, and only the latter accounts for a threshold-current density (by assuming a saturation effect). If it is true, as indicated in this paper, that the magnetic effect is the more fundamental, it would seem that this fact might afford a valuable clue leading toward a more satisfactory theory of the superconducting state and perhaps of metallic conduction in general.

Washington, February 12, I9I 7.

\footnotetext{
9 Onnes, Leiden Comm. No. IIg.

${ }^{10}$ Lindemann, F. C., Phil. MIag., 29, p. 127; 1915.

11 Thomson, J. J., Phil. Mag., 30, p. 192; 1915.
} 